\title{
The Broken-Point Continuingly-Transferring Design Based on Satellite Network
}

\author{
Jianmin Mei ${ }^{1}$, Guiqiang $\mathrm{Ni}^{1,{ }^{1}}$, Jinsong Jiang ${ }^{1}$, Yingzi Yan $^{1}$, Qing Liu ${ }^{1}$ and Junxian Long ${ }^{2}$ \\ ${ }^{1}$ Institute of Command Information System, PLA University of Science and Technology, Nanjing 210007, China \\ ${ }^{2}$ Chinese People’s Liberation Uint 69066, Wulumuqi 830092, China \\ ${ }^{*}$ Corresponding author
}

\begin{abstract}
High channel bit error rate, long delay and other characteristics of the satellite network, will cause the file in the process of transmission is easy to interrupt or packet loss. In order to improve the efficiency of file transfer, the server sends data once, while multiple earth station clients receive., and proposed broken-point continuingly-transgerring mechanism based on satellite network, this mechanism will enable the earth station just accept the unreceived data, which greatly improves the efficiency of file transfer.
\end{abstract}

Keywords-satellite network; file transfer; earth station; brokenpoint continuingly-transferring

\section{INTRODUCTION}

In satellite communication networks, with soft increasing proportion in the earth station, files assume more and more features. File transmission mechanism has a massive impact on the file transfer efficiency in the earth station. broken-point continuingly-transferring mechanism of satellite network file transfer is one of the important mechanisms.

The high bit error rate, long transmission delay [1] and easily affected by natural conditions, will cause the instability of the satellite network, which often leads to the instability of the data transmission [2]. In the process of data transmission, it will lead to the failure of data transmission if the network connection is interrupted or due to power outages, natural disasters and other factors lead to the termination of the program. Breakpoint continuingly technology of data frame is based on file transfer. It is a technology that can save the data that has been successfully transmitted, and will connect again at a certain time to transfer remaining data. It can save network traffic and improve the transmission efficiency.

The satellite network file transfer often send data in the form of broadcast, which achieve file download server sends the file once, a plurality of earth stations receive it at the same time. This approach can effectively save the precious satellite network bandwidth in normal reception case, and can improve the efficiency of file transfer. However, the state of satellite network link is unstable and link interruption may occur in the process of one or more the earth stations receiving a file. These earth stations will send requests to file server after the failure of receiving this file, then the server will broadcast again and again. Finally, all the earth stations can successfully receive this file, which may transfer many times.

\section{RELATED WORK}

File transfer mechanism originated in the Internet at first, FTP protocol can provide efficient and reliable data transmission services [3], it can transfer files through the interconnection of heterogeneous computers [4]. Then file transfer matures up in a variety of Internet applications. In the satellite network, there are some scholars studying the file transfer system based on the satellite network, and paper [5] applies the selective retransmission mechanism based on error recovery to the satellite network file transmission system. Due to the low bandwidth rate, long time delay and high packet loss rate of the satellite network, paper [6] proposes ARSFTP transmission protocol to solve the problem of file transmission in satellite network.

Broken-point continuingly-transferring mechanism is based on the file transfer. Based on FTP protocol and Http protocol broken-point continuingly-transferring mechanism is very mature. Paper [7] develops file transfer system supporting broken-point continuingly-transferring on the basis of file transfer system. In order to solve data repeat transmission because of interruption, which results in the waste of time and resource, paper [8] proposes broken-point continuinglytransferring mechanism based on tree structure. These mechanism are all based on point-to-point transmission technology, are not suitable for using in satellite network down channel broadcast environment. Therefore, the satellite network based on broken-point continuingly-transferring mechanism is very necessary.

\section{SYSTEM ARCHITECTURE}

In satellite communication system, because of the up and down channel asymmetry, file transmission system using broadcast mode, achieves file download server sends the file once, a plurality of earth stations receive it at the same time. The server send file data frame via Send Antenna, and broadcast to terminal devices, these include vehicle Terminal, PDA, Fixed Access Device and so on, the satellite communication system architecture is shown in Figure I.

\section{A. File Transfer Process Design}

According to paper [9], the server model consists of three parts: file blocking, frames construct and broadcast file data frame. The client model also consists of three parts: receive file data frame, devide frame into block and composite file. The function of each part is shown in Table I. 


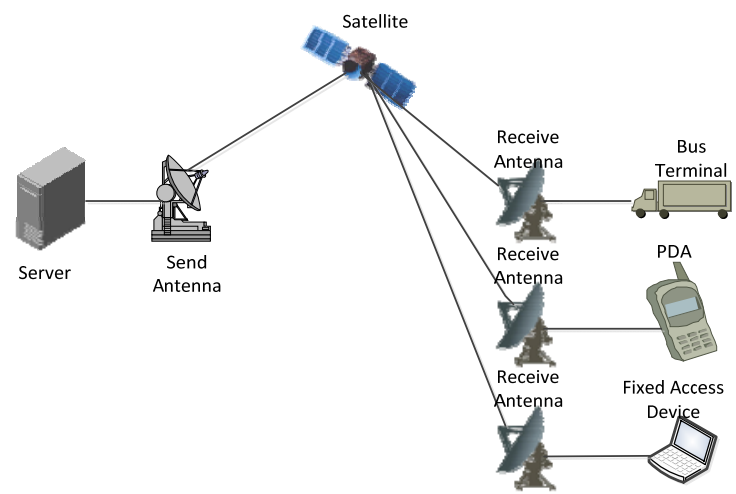

FIGURE I. SATELLITE COMMUNICATION SYSTEM ARCHITECTURE

In the design of the file transfer process, the server sends the file data, the earth station terminal equipment receives the file data, the sending and receiving reverses each other. The file transfer process is shown in Figure II.

TABLE I. SEND AND RECEIVE FILE DATA OPERATION

\begin{tabular}{|l|l|l|}
\hline & \multicolumn{1}{|c|}{ Operation } & \multicolumn{1}{|c|}{ Description } \\
\hline Server & file blocking & Devide the file into equal blocks \\
\cline { 2 - 3 } & construct frame & $\begin{array}{l}\text { construct a frame with head and file } \\
\text { blocking,and input send queue }\end{array}$ \\
\cline { 2 - 4 } & $\begin{array}{l}\text { Broadcast data } \\
\text { frame }\end{array}$ & $\begin{array}{l}\text { Read and broadcast data from the } \\
\text { sending queue }\end{array}$ \\
\hline Client & $\begin{array}{l}\text { Data received } \\
\text { frame }\end{array}$ & $\begin{array}{l}\text { Receiving data and input receive } \\
\text { queue }\end{array}$ \\
\cline { 2 - 4 } & $\begin{array}{l}\text { Devide into } \\
\text { blocks }\end{array}$ & $\begin{array}{l}\text { Read queue elements, and get the file } \\
\text { bloching }\end{array}$ \\
\cline { 2 - 3 } & Composite file & $\begin{array}{l}\text { Write the obtained blocks to } \\
\text { corresponding position. }\end{array}$ \\
\hline
\end{tabular}

B. Broken-Point Continuingly-Transferring File Transfer Protocol

In this design, the server includes file broadcast frame, file information frame and file check frame, the client mainly includes file request frame, broken-point continuinglytransferring frame and file finished frame. The frame function of each protocol is shown in Table II.

TABLE II. FILE TRANSFER PROTOCOL TABLE

\begin{tabular}{|c|c|c|}
\hline & Protocol & Description \\
\hline \multirow{3}{*}{ Server } & file broadcast frame & $\begin{array}{l}\text { broadcast the file name to the } \\
\text { client }\end{array}$ \\
\hline & $\begin{array}{l}\text { file information } \\
\text { frame }\end{array}$ & file data send to the client \\
\hline & file check frame & construct a MD5 Frame \\
\hline \multirow{3}{*}{ Client } & file request frame & request the broadcast file name \\
\hline & $\begin{array}{l}\text { broken-point } \\
\text { continuingly- } \\
\text { transferring frame }\end{array}$ & $\begin{array}{l}\begin{array}{l}\text { input the lack frame to the } \\
\text { broken-point } \\
\text { transferring } \\
\text { transferring frame }\end{array} \\
\text { continuingly- } \\
\end{array}$ \\
\hline & file finished frame & sign the client receives finished \\
\hline
\end{tabular}

On initial conditions, the server sends file broadcast frame, and then the earth station sends the file request frame. When the server receives the request, it will send all file information frame and then send file check frame. The client calculates whether there are missing frames, if not the file download is complete, then sends file finished frame to the server. If there is a loss, sending broken-point continuingly-transferring frame to the server, the server receives the corresponding brokenpoint continuingly-transferring request, then broadcast the requires data to the earth station, until all the earth station's application has been completed. Protocol interaction flow diagram is shown in Figure IV.

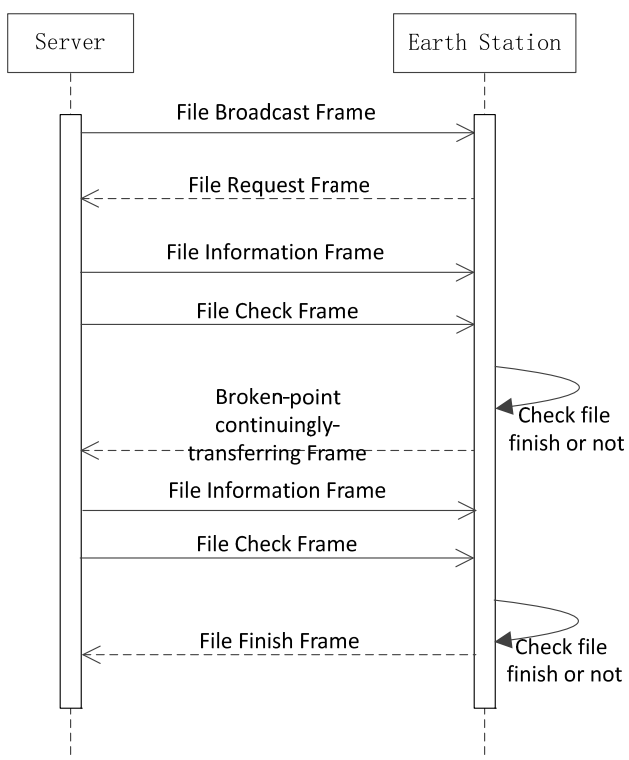

FIGURE II. THE PROTOCOL OF FILE TRANSPORT WITH SERVER AND CLIENT COMMUNICATION

\section{The Algorithm Of Broken-Point Continuingly- TRANSFERRING}

\section{A. Interrupt Definition}

Broken-point continuingly-transferring can be divided into two parts to understand, the first is the perception of a breakpoint, and the second is resume strategy. A breakpoint means a file that divided into several parts in the process of downloading. According to the satellite channel, this phenomenon may be caused by abnormal program termination factors of transmission quality of space channel or by electricity, such as natural disasters. The former cause of the interrupt is called network interruption, and the latter causes called host interrupt. Resume is a strategy that can be used when interrupt occurred, which recovers the transmission from breakpoint until the document is received.

\section{B. Interrupt Detection Strategy}

Interrupt detection strategy based on state change: In the satellite network file transfer system, the status of earth stations is divided into INIT_STATUS and IN_DOWNLOAD, the state of file $\mathrm{F}$ is divided into UNDOWNLOAD and DOWNLOAD. When earth station of the satellite communication network is in downloading procedure with file $\mathrm{F}$, if the download procedure is not finished while receiving waiting time has exceeded the timeout, the state of earth station change from download status into a starting state, at 
this time, the file $\mathrm{F}$ in the download status, the earth station status is INIT_STATUS, then the file F's status is DOWNLOAD, then the earth station has broken.

Interrupt detection strategy based on file size: When the state of earth station is INIT_STATUS, we can detect interrupt by verify whether the received frame is equal to the total number of files, if not, the download procedure is interrupted.

\section{Resume Strategy}

When the earth station detects interrupt, sending Brokenpoint Continuingly-transferring request frame, which contains the information that earth station needed, the frame number can be obtained from memory, and described as the lack frame number segment. If the host interrupt occurs, all the missing consecutive frame serial number is recovered from the configuration file to the memory, and then the missing segment is obtained from the memory.

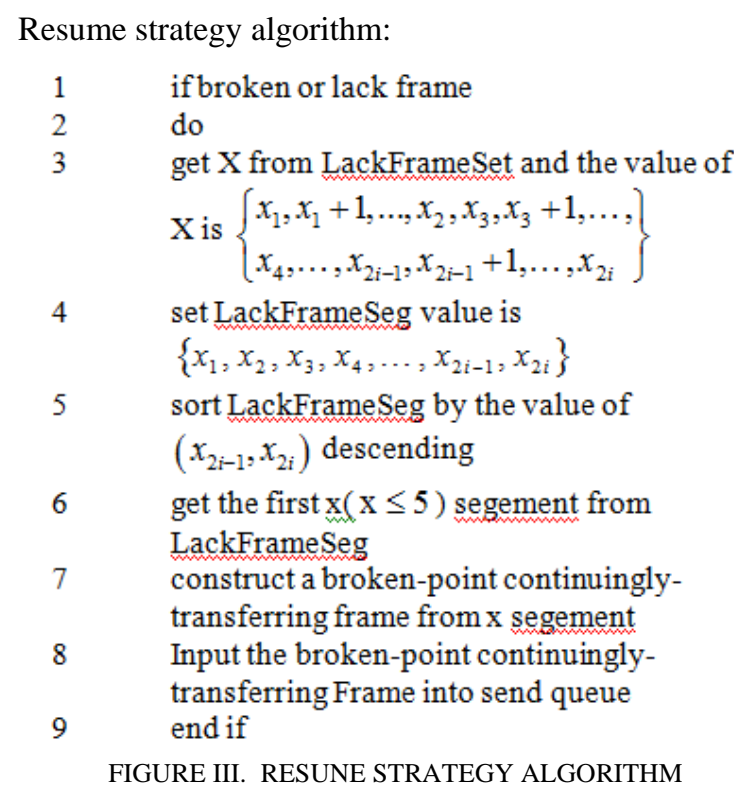

A configuration file is necessary in Broken-point Continuingly-transferring mechanism, containing the station type, station address, file name, status of earth station, and the lack frame segment. One station address is mapped to one earth station, and the rest of the field is used to mark the information of the software receiving.

If the host interrupt occurs during the file reception, the software information is recovered from the configuration file, and the method to update the software information in the configuration file is:

1) Initialize to receive the file $F$, missing all the serial number, recorded as 1-total;

2) If the serial number of the frame is received, the serial number of the received frame will be updated;

3) If the serial number is equal to 20 frames, the frame number in the update configuration file will be updated.

Throughout the transmission cycle earth stations and file status changes as shown in TableIII:
TABLE III. STATUS CHANGE IN A PERIOD OF TIME

\begin{tabular}{|c|c|c|}
\hline Condition & Earth Station Status & File Status \\
\hline Not Receive & INIT_STATUS & UNDOWNLOAD \\
\hline Begin Receive & IN_DOWNLOAD & DOWNLOAD \\
\hline Broken & INIT_STATUS & DOWNLOAD \\
\hline Finish Receive & INIT_STATUS & UNDOWNLOAD \\
\hline
\end{tabular}

\section{The Algorithm Of BROKEn-PoInt ContinUingly- TRANSFERRING}

Let the probability of this $\mathrm{n}$ earth station interrupt as $\mathrm{p}$, frame sending interval for file download server as $\mathrm{T}$, Channel bandwidth as $\mathrm{W}$ kbps, frame length of each frame as L Byte, theoretically total frame number of file as $\mathrm{N}$, frame transmission interval is $\mathrm{T}=(\mathrm{L} * 8) /(\mathrm{W} * 1024)$, unit for seconds. Actually it is often make the corresponding adjustment based on the quality of the channel. That is a constant c on the T

$$
\mathrm{T}=\mathrm{C} *(\mathrm{~L} * 8) /(\mathrm{W} * 1024)
$$

File download server sends files to the $\mathrm{n}$ earth station agents at the same time. The quantitative indicator of this analysis is the time spent by the server to complete the application of all earth stations. The cost of file transfer system without using the breakpoint continuingly mechanism is $T_{\text {old,i }}$. The cost of file transfer system using the breakpoint continuingly mechanism is $\mathrm{T}_{\text {new, } \mathrm{i}}$.The time of the retransmission of lost packets is ignored.

Let us suppose the interrupts of the $\mathrm{k}$ earth stations at the same time as one interrupt. When interrupt occur at time $\mathrm{i}$, the server sent a total of $\mathrm{x}_{\mathrm{i}}$ frames $\left(\mathrm{x}_{0}=0\right)$. Set the $\mathrm{k}(0 \leq \mathrm{k} \leq \mathrm{n})$ earth station interrupts occur at the same time, which separately received $\mathrm{X}_{\mathrm{i} 1}, \mathrm{x}_{\mathrm{i} 2}, \ldots, \mathrm{X}_{\mathrm{ik}}\left(0 \leq \mathrm{x}_{\mathrm{ij}} \leq \mathrm{x}_{\mathrm{i}}, 1 \leq \mathrm{j} \leq \mathrm{k}\right)$ frames. Because of interrupt occurs in download process, the waiting time of server does not affect analysis. Set the waiting time of server interrupt occurred at time i as $\mathrm{T}_{\text {wait,i }}$.

When an interrupt occurs, the cost of file transfer system without using the breakpoint continuingly mechanism is:

$$
\begin{aligned}
& \mathrm{T}_{\text {old }, 1}=C_{n}^{0}(1-p)^{n} \mathrm{NT}+C_{n}^{1} \mathrm{p}(1-p)^{n-1}\left(\mathrm{x}_{1}+\mathrm{N}\right) \mathrm{T} \\
& +C_{n}^{2} \mathrm{p}^{2}(1-p)^{n-2}\left(\mathrm{x}_{2}+\mathrm{N}\right) \mathrm{T}+\ldots+C_{n}^{n} \mathrm{p}^{n}\left(\mathrm{x}_{\mathrm{n}}+\mathrm{N}\right) \mathrm{T} \\
& +\mathrm{T}_{\text {wait }, 1}
\end{aligned}
$$

The cost of file transfer system using the breakpoint continuingly mechanism is:

$$
\begin{aligned}
& \mathrm{T}_{\text {new }, 1}=C_{n}^{0}(1-p)^{n} \mathrm{NT}+\mathrm{C}_{h}^{1} \mathrm{p}(1-p)^{n-1}\left(\mathrm{x}_{11}+\mathrm{N}\right) \mathrm{T} \\
& +C_{n}^{2} \mathrm{p}^{2}(1-p)^{n-2}\left(\max \left(\mathrm{x}_{11}, \mathrm{x}_{12}\right)+\mathrm{N}\right) \mathrm{T}+\ldots+ \\
& C_{n}^{n} \mathrm{p}^{n}\left(\max \left(\mathrm{x}_{11}, \mathrm{x}_{12}, \ldots, \mathrm{x}_{1 \mathrm{n}}\right)+\mathrm{N}\right) \mathrm{T}+\mathrm{T}_{\text {wait }, 1}
\end{aligned}
$$


Formula (2), (3) can be simplified by,

$$
\begin{aligned}
\mathrm{T}_{\mathrm{old}, 1} & =T \sum_{i=1}^{n} \mathrm{x}_{\mathrm{i}}+N \mathrm{Tnp}(1-\mathrm{p})+\mathrm{T}_{\text {wait }, 1} \\
\mathrm{~T}_{\text {new }, 1} & =T \sum_{i=1}^{n} \max \left\{\mathrm{x}_{1 \mathrm{i}}\right\}+N \operatorname{Tnp}(1-\mathrm{p})+\mathrm{T}_{\text {wait }, 1}
\end{aligned}
$$

From the above analysis and simplification we can deduce when interrupt occur at times $\mathrm{k}$ :

$$
\begin{aligned}
\mathrm{T}_{\text {old }, \mathrm{k}}=k \mathrm{~T}_{\text {old }, 1} & =k T \sum_{i=1}^{n} \mathrm{x}_{\mathrm{i}}+k N \operatorname{Tnp}(1-\mathrm{p})+\sum_{i=1}^{k} \mathrm{~T}_{\text {wait }, i} \\
\mathrm{~T}_{\text {new }, \mathrm{k}} & \approx \mathrm{TN}+k N \operatorname{Tnp}(1-\mathrm{p})+\sum_{i=1}^{k} \mathrm{~T}_{\text {wait }, i}
\end{aligned}
$$

When the interrupt occurs at time $\mathrm{k}$, file is about to receive the completion. Now $\mathrm{x}_{\mathrm{i}} \approx \mathrm{N}(1 \leq \mathrm{i} \leq \mathrm{n})$, In case that earth station client reconnect when the server is still broadcasting the information frame, that is $\mathrm{T}_{\text {wait, } \mathrm{i}}=0(1 \leq \mathrm{i} \leq \mathrm{k})$. Now formula (6) and (7) can be simplified .

$$
\begin{gathered}
\mathrm{T}_{\text {old }, \mathrm{k}}=k \operatorname{TnN}+\operatorname{kTnNp}(1-\mathrm{p}) \\
\mathrm{T}_{\text {new }, \mathrm{k}}=\operatorname{TN}+\mathrm{kNTnp}(1-\mathrm{p}) \\
\lim _{k \cdot n \rightarrow \infty} \frac{\mathrm{T}_{\text {new }, \mathrm{k}}}{\mathrm{T}_{\text {old }, \mathrm{k}}}=\lim _{k \cdot \mathrm{n} \rightarrow \infty} \frac{k n\left(1+p-p^{2}\right)}{(1+\mathrm{knp}(1-\mathrm{p}))} \approx \frac{1+p}{\mathrm{p}}=1+\frac{1}{\mathrm{p}}
\end{gathered}
$$

By the conclusions of formula (10), when the number of earth stations in the satellite network is more than enough and the number of interrupt times reach a certain amount, we can say that file transfer using breakpoint continuingly mechanism than not using this mechanism in high efficiency $1 / p$ times. Usually $\mathrm{P}$ is a smaller number.

\section{CONCLUSION}

Socket programming uses the boost library to achieve design and implementation the broken-point continuinglytransferring based on satellite network, is developed by using interrupt sensing strategy based on the change of state, and ensures the implementation of interrupt mechanism in breakpoint continuingly include host interrupts and network interrupts. In the simulation environment, the cable to unplug the human timeout and then plug in, the earth station client can continue normal transfer, the file can be downloaded completely. If restarting the client after powering off it artificially, the client can do the same. This method can be applied to the satellite network with low service rate, and it can realize the nonsense of service rate. In the case of poor quality of the channel, the reliability and efficiency of the file transfer on the space channel can be guaranteed, and the efficiency and quality of the file transmission on the space channel can be improved.

\section{REFERENCES}

[1] Wan G, Liu G, Liu P. UDP-based Reliable Data Transfer Protocol for Satellite Networks[J]. Information Security \& Communications Privacy, 2007.

[2] ZHOU X, RUAN S. Implementation of Breakpoint continuingly Based on Multi-threaded $[\mathrm{J}][\mathrm{J}]$. Computer Knowledge and Technology (Academic Exchange), 2007, 22: 043

[3] Reynolds J, Reynolds J. Rfc959 file transfer protocol (ftp)[J]. www, 1985, 2(78):312-319.

[4] Gien M. A File Transfer Protocol (FTP)[J]. Computer Networks, 1978, 2(4-5):312-319.

[5] Henriksen E, Aas G, Rydningen J B. A transport protocol supporting multicast file transfer over satellite links[C]// Eleventh International Phoenix Conference on Computers and Communications, 1992. Conference Proceedings. 1992:590-596.

[6] Morrison, Andrew. Amateur Radio Satellite File Transfer Protocol (ARSFTP)[J]. 2012.

[7] Chen Z, Guo J, Liu Z, et al. Design and Key Technology of the Filetransfer System with the Function of broken-point continuinglytransferring[J]. Computer Engineering, 2002, 28(12):14-16.

[8] Xiao Z Y. Pipelined dissemination and broken-point continuinglytransferring technologies for CAPF oriented data transmission application[D]. National University of Defense Technology, 2009.

[9] WEI XiaoLi, DUAN Tao. Design and Implementation of a Reliable Transport Protocol for the Satellite Broadcasting Environment [J]. Computer Systems \& Applications, 2010, 19(1):63-66.

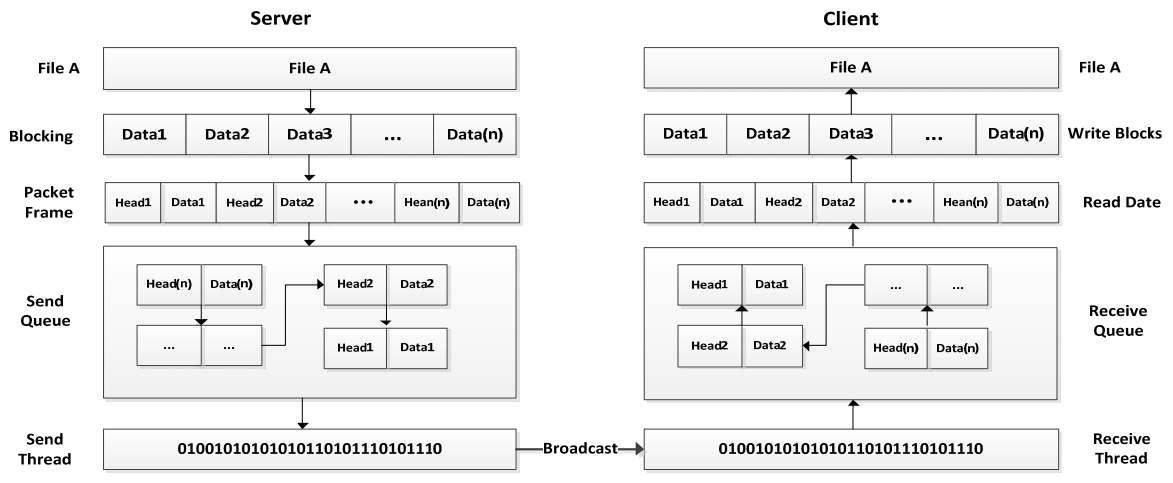

